\title{
Mumps in a 27-year-old man
}

\author{
J. Brad Wiggers MD, Tiffany Chan MD, Wayne L. Gold MD, Derek R. MacFadden MD
}

Cite as: CMAJ 2017 April 18;189:E569-71. doi: 10.1503/cmaj.161347

A 27-year-old previously healthy man presented to the emergency department with a two-day history of fever and right-sided scrotal swelling. He was in a monogamous relationship with a male partner and had no history of HIV or other sexually transmitted infections. He had immigrated to Canada from China five years prior, and records of his childhood vaccinations included immunization against measles-mumpsrubella (MMR) but did not specify the number of doses.

The patient reported that he had a self-limited episode of bilateral painful neck swelling seven days before presenting to the emergency department. He did not have headache, neck stiffness, abdominal pain, urinary tract symptoms, urethral discharge or rash. Notably, his partner had symptoms of bilateral parotid gland enlargement about two weeks before the onset of his illness.

Upon examination, his temperature was $39.0^{\circ} \mathrm{C}$. The oropharynx was normal, and there was no meningismus or swelling of the parotid glands. Testicular examination showed a tender right testis and epididymis.

Laboratory investigations showed that the patient had a normal complete blood cell count and a serum lipase level of $22 \mathrm{U} / \mathrm{L}$ (normal $\leq 60 \mathrm{U} / \mathrm{L}$ ). Initial investigations included a throat swab for group A streptococcus and a nasopharyngeal swab to detect respiratory viruses using multiplex polymerase chain reaction testing. He had blood taken for cytomegalovirus immunoglobulin M (IgM) antibody, a monospot test, parvovirus B19 IgM antibody and testing for HIV. He also had nucleic acid amplification testing of urine and testing of pharyngeal swabs for both Neiserria gonorrhoeae and Chlamydia trachomatis. Scrotal ultrasonography showed an enlarged hyperemic right testicle (measuring $5.1 \times 3.3 \times 3.3 \mathrm{~cm}^{3}$ ) and an edematous hyperemic epididymis. Given the constellation of fever, prior neck swelling, right epididymo-orchitis and history of bilateral parotid swelling in his partner, serum mumps serologic testing and polymerase chain reaction testing of both urine and buccal samples for mumps virus were requested.

Two days later, he was seen at follow-up in the infectious disease clinic. His fever had resolved, and the swelling and pain in his scrotum had improved. Results from tests on the nasopharyngeal specimen were negative for influenza $A$ and $B$, and respiratory syncytial virus. Results from urine polymerase chain reaction testing were positive for mumps, and the patient had an elevated mumps IgM antibody level. Polymerase chain reaction testing of the nasopharyngeal swab for mumps was performed, and the result was also positive. Results of other microbiologic

\section{KEY POINTS}

- Mumps is a viral illness that classically causes bilateral parotitis; extrasalivary manifestations may include epididymo-orchitis, oophoritis, pancreatitis and aseptic meningitis.

- Canadians born between 1970 and 1992 are at increased risk of mumps because they lack natural immunity and received only one dose of vaccine in their childhood immunization series.

- Outbreaks continue to occur in North America, even among highly vaccinated populations, which may be the result of both primary vaccine failure and waning immunity.

- The test of choice for mumps diagnosis is a reverse transcription-polymerase chain reaction testing of a buccal swab; serology is not useful in vaccinated populations.

- No testing is necessary in patients with a compatible clinical syndrome who are linked to a confirmed case of mumps.

investigations were negative. Contact tracing by the local public health unit was conducted, and no additional active cases were identified.

\section{Discussion}

Mumps is a contagious viral illness for which humans are the only natural host. ${ }^{1}$ The virus circulated widely in Canada before the introduction of a live attenuated vaccine in 1969. People born before 1970 are presumed to have developed natural immunity. ${ }^{1}$ Because MMR vaccination was introduced as part of routine childhood immunization, most cases now occur among adults 20 years of age and older. ${ }^{1}$ Canadians born between 1970 and 1992 are susceptible because their vaccine schedule included only one dose and they lack natural immunity. ${ }^{2}$ Given the age of this patient, it is likely that he would have received only one dose of vaccine.

\section{Clinical features}

Mumps is transmitted either through droplet spread or direct contact with the saliva of an infected person. ${ }^{1}$ The incubation period is 15 to 24 days, ${ }^{2}$ and patients are considered to be contagious from two days before to five days after the onset of parotitis. ${ }^{1}$ About one-third of infections are asymptomatic. ${ }^{3}$

Parotitis is the hallmark of mumps, occurring in $95 \%$ of patients with symptomatic disease, and is bilateral in most cases. ${ }^{3}$ It typically 
begins unilaterally, with involvement of the contralateral parotid gland within several days. Other salivary glands are less commonly affected. Most patients have a brief prodrome of fever, malaise, anorexia and headache before the onset of parotitis. ${ }^{3}$ Our patient's illness began with fever and painful bilateral neck swelling, which had resolved by the time of our assessment. With microbiologic confirmation of mumps, it is likely that the patient was experiencing parotitis. However, it is possible that he was experiencing submandibular sialadenitis, which may mimic anterior cervical lymphadenopathy.

Epididymo-orchitis is the most common extrasalivary manifestation, occurring in 15\%-30\% of postpubertal men. ${ }^{3}$ Orchitis is bilateral in about one-quarter of cases and develops four to eight days after parotitis, as in our patient. Infertility secondary to orchitis is uncommon; however, reduction in testicle size may occur in $50 \%$ of patients and abnormal results for sperm testing in $25 \% .^{3}$

Uncommon extrasalivary manifestations include oophoritis (5\%), aseptic meningitis ( $1 \%-10 \%)$, transient hearing loss $(4.1 \%)$ and encephalitis $(0.1 \%){ }^{3}$ Death (1.5\% of cases of encephalitis) and permanent hearing loss $(0.005 \%)$ are uncommon. Pancreatitis can also occur, but the normal result for serum lipase ruled this out in our patient.

\section{Diagnosis}

The presence of parotitis should prompt physicians to consider a diagnosis of mumps. Although the diagnosis may be clear in an outbreak setting, a variety of other infectious and noninfectious etiologies can cause parotid enlargement (Box 1). Features of these conditions should be obtained through a detailed history, physical examination and directed investigations. Historical features supporting an alternate diagnosis include radiation exposure (primary salivary neoplasm), known primary malignant disease (metastasis), symptoms of a systemic autoimmune condition (Sjögren syndrome or sarcoidosis), unilateral suppurative parotitis (bacterial) or use of drugs known to cause parotid enlargement/parotitis. ${ }^{3-6}$ Many other viruses are associated with parotitis. Epstein-Barr virus is a common cause of mumps-like illness in nonoutbreak settings. ${ }^{3}$ Mumps orchitis should be considered in acute testicular pain, which can also be caused by testicular torsion, epididymitis, Fournier gangrene and appendiceal torsion.

In an outbreak setting, the Public Health Agency of Canada advises against the use of diagnostic tests. The diagnosis of a "confirmed case" can be made clinically based on a mumpscompatible illness with an epidemiologic link to a laboratoryconfirmed case. ${ }^{7}$ For sporadic cases, buccal swab polymerase chain reaction is considered the test of choice and should be collected ideally within three to five days of symptom onset. ${ }^{7}$ Dacron-, nylon- and rayon-tipped swabs are appropriate and should be placed in a viral transport medium. ${ }^{2}$

Results for polymerase chain reaction testing of urine may be positive up to 14 days after symptom onset, but it has been shown to be less sensitive than buccal samples in recent outbreaks. ${ }^{7}$

Serologic testing is available, but IgM has been shown to lack sensitivity in outbreaks that included partially vaccinated populations. ${ }^{7}$ Additionally, mumps IgM is nonspecific; therefore, a pos-
Box 1: Differential diagnosis for enlargement of the parotid gland ${ }^{3-6}$

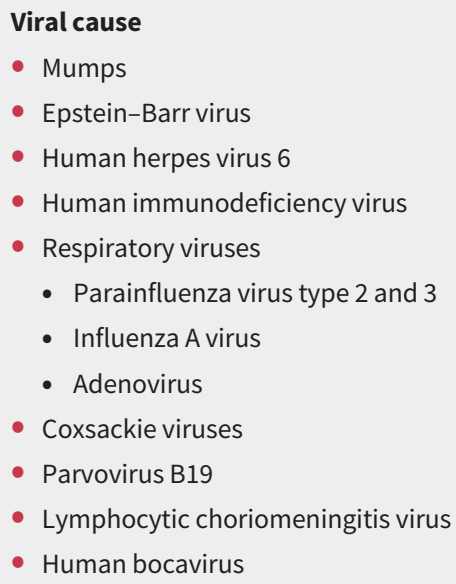

\section{Bacterial cause}

- Staphylococcus aureus

- Oral streptococci and oral anaerobes

- Gram-negative bacteria (including Burkholderia pseudomallei)

- Mycobacterium tuberculosis

- Non-tuberculous mycobacteria

\section{Autoimmune disorders}

- Sjögren syndrome

- Sarcoidosis

\section{Neoplastic disorders}

- Primary salivary gland neoplasm

- Lymphoma

- Metastatic malignant disease

Drugs

- Propylthiouracil

- Phenothiazines

- lodides

- Phenylbutazone

\section{Other conditions}
- Sialolithiasis
- Malnutrition
- Chronic alcoholism
- Uremia
- Diabetes mellitus
- Cirrhosis
- Anorexia nervosa

itive result for IgM antibody without a link to a confirmed case could represent a false-positive result. ${ }^{7} \mathrm{~A}$ fourfold increase in mumps IgG antibody levels between acute and convalescent sera is considered diagnostic of acute mumps infection. ${ }^{7}$

\section{Management and prevention}

Mumps is a self-limited illness for which no specific treatment is required. Supportive care with analgesics for parotitis or orchitis may be required. Immunization with a vaccine containing a live 
attenuated mumps component is the main strategy for preventing infection and clinical disease. Since the introduction of the single-dose mumps vaccine in 1969, there has been a $99 \%$ decline in mumps cases, with a further decrease seen after the introduction of the two-dose vaccine schedule in 1996/97. ${ }^{1}$ The current Canadian immunization schedule recommends administration of the first mumps-containing vaccine dose at 12 to 15 months and the second at 18 months. ${ }^{1}$ However, provincial schedules and vaccine usage vary and should be confirmed with local authorities. Pregnancy, prior anaphylaxis to the vaccination, anaphylaxis to any component of the vaccine with the exception of eggs and immunocompromised status are contraindications to immunization. ${ }^{1}$

Despite the success of universal childhood immunization programs, mumps outbreaks continue to occur in North America. Both primary vaccination failure and waning immunity are thought to play a role. ${ }^{7}$ In a 2009/10 outbreak in Ontario involving 134 patients, $69.5 \%$ were incompletely immunized. ${ }^{2}$ However, a 2015/16 outbreak at the University of Illinois involving 317 persons (32\% confirmed and $68 \%$ probable cases) occurred in a highly vaccinated population, with $89 \%$ of patients having received at least two doses of the MMR vaccine. ${ }^{8}$ The effectiveness of mumps vaccination ranges from $62 \%-91 \%$ after one dose to $76 \%-91 \%$ after two doses. ${ }^{1}$ Recommendations for vaccination of individuals with incomplete vaccination schedules who were born between 1970 and 1992, including health care workers, university students living in residence, military personnel and international travellers to endemic areas, can be found in the Canadian Immunization Guide. ${ }^{1}$

Mumps is a communicable disease and a notifiable illness. The true incidence in Canada may be underestimated: a crossCanada survey showed that only 59\% of emergency medicine physicians knew that mumps was reportable. ${ }^{9}$ Inpatients should be placed under droplet precautions, and outpatients should be excluded from school or work for five days after onset of parotitis. Additional infection control measures can be found in the Guideline for the prevention and control of mumps outbreaks in Canada. ${ }^{7}$

\section{Conclusion}

Since the introduction of a universal immunization program in Canada, the epidemiology of mumps has changed. Adults aged 20 years or older now represent most cases. Our patient may have received only one dose of vaccine, which would put him in the same at-risk group as those born in Canada between 1970 and 1992. Physicians should consider a diagnosis of mumps in adults with a compatible clinical illness, and notify local public health units accordingly.

\section{References}

1. Canadian Immunization Guide: Part 4 - Active vaccines: mumps vaccine. Ottawa: Public Health Agency of Canada; 2012. Available: http://healthy canadians.gc.ca/publications/healthy-living-vie-saine/4-canadian-immunization -guide-canadien-immunisation/index-eng.php?page=14\#ru (accessed 2016 Sept. 8).

2. Deeks SL, Lim GH, Simpson MA, et al. An assessment of mumps vaccine effectiveness by dose during an outbreak in Canada. CMAJ 2011;183:1014-20.

3. Hviid A, Rubin S, Mühlemann K. Mumps. Lancet 2008;371:932-44.

4. Mehanna $\mathrm{H}, \mathrm{McQueen} \mathrm{A}$, Robinson $\mathrm{M}$, et al. Salivary gland swellings. BMJ 2012;345:e6794.

5. White NJ. Melioidosis. Lancet 2003;361:1715-22.

6. Barskey $\mathrm{AE}$, Juieng $\mathrm{P}$, Whitaker $\mathrm{BL}$, et al. Viruses detected among sporadic cases of parotitis, United States, 2009-2011. J Infect Dis 2013;208:1979-86.

7. Guidelines for the prevention and control of mumps outbreaks in Canada. Can Commun Dis Rep 2010:36(Suppl):1-46. Available: www.phac-aspc.gc.ca/publicat/ ccdr-rmtc/10pdf/36s1-eng.pdf (accessed 2017 Feb. 2).

8. Albertson JP, Clegg WJ, Reid HD, et al. Mumps outbreak at a university and recommendation for a third dose of Measles-Mumps-Rubella vaccine - Illinois, 2015-2016. MMWR Morb Mortal Wkly Rep 2016;65:731-4.

9. Friedman SM, Sommersall LA, Gardam M, et al. Suboptimal reporting of notifiable diseases in Canadian emergency departments: a survey of emergency physician knowledge, practices and perceived barriers. Can Commun Dis Rep 2006;32:187-98. Available: www.phac-aspc.gc.ca/publicat/ccdr-rmtc/06vol32/ dr3217a-eng.php (accessed 2017 Feb. 2).

\section{Competing interests: None declared.}

This article has been peer reviewed.

The authors have obtained patient consent.

Affiliation: Division of Infectious Diseases, Department of Medicine, University of Toronto, Toronto, Ont.

Contributors: All of the authors had access to the data, contributed to the preparation of the manuscript and revised it critically for important intellectual content, approved the final version for publication and agreed to be accountable for all aspects of the work.

Correspondence to: Derek MacFadden, derek.macfadden@uhn.ca

The section Cases presents brief case reports that convey clear, practical lessons. Preference is given to common presentations of important rare conditions, and important unusual presentations of common problems. Articles start with a case presentation (500 words maximum), and a discussion of the underlying condition follows (1000 words maximum). Visual elements (e.g., tables of the differential diagnosis, clinical features or diagnostic approach) are encouraged. Consent from patients for publication of their story is a necessity. See information for authors at www.cmaj.ca. 